\title{
Effects of cytomegalovirus infection on the prognosis of inflammatory bowel disease patients
}

\author{
WEI-XIA ZHANG, CHENG-YAN MA, JIAN-GUO ZHANG, FENG HE, QING-MIN LIU, AIBIN CHENG, \\ TIEJUN LIU, JUNWEI ZHANG, JIANJUN WANG, XUAN BU, YUXI XIE, ZENGLI DIAO and JING BAI
}

Department of Critical Care Medicine, Linyi People's Hospital, Linyi, Shandong 276003, P.R. China

Received July 14, 2015; Accepted September 6, 2016

DOI: $10.3892 / \mathrm{etm} .2016 .3763$

\begin{abstract}
The aim of the present study was to investigate the effects of cytomegalovirus (CMV) infection on the prognosis of inflammatory bowel disease (IBD). Various databases were searched using a combination of keywords associated with CMV infection and IBD. Subsequent to the selection of relevant studies in line with strict inclusion and exclusion criteria, a meta-analysis was conducted using the Stata 12.0 software. A total of 195 studies were initially retrieved, including 28 studies in Chinese and 167 in English. Following the exclusion of unsuitable studies, 7 cohort studies with 374 IBD patients were included in the meta-analysis. The results of the present study identified significant differences between patients with and without CMV infection regarding the disease duration of IBD [standardized mean difference, $-0.81 ; 95 \%$ confidence interval $(\mathrm{CI}),-1.19$ to $-0.43 ; \mathrm{P}<0.001]$, the efficacy of corticosteroid therapy [relative risk (RR), $1.24 ; 95 \% \mathrm{CI}, 1.02-1.49 ; \mathrm{P}=0.029]$, the colectomy rate $(\mathrm{RR}$, 2.13; $95 \% \mathrm{CI}, 1.03-4.40 ; \mathrm{P}=0.042)$ and the incidence of severe IBD (RR, 1.32; 95\% CI, 1.04-1.67; P=0.022). Considering the IBD onset area, patients with CMV infection may have higher susceptibility to pancolitis (RR, 1.31; 95\% CI; 1.01-1.72; $\mathrm{P}=0.045$ ); however, no difference in susceptibility to left-sided IBD was observed between patients with or without CMV infection ( $R R, 0.97$; 95\% CI, 0.72-1.30; $\mathrm{P}=0.828$ ). In conclusion, CMV infection may be associated with the disease duration, efficacy of corticosteroid therapy, colectomy rate, severe IBD incidence and disease location of IBD; thus, the presence of CMV infection may be considered as an important biomarker for determining the prognosis of IBD.
\end{abstract}

Correspondence to: Dr Cheng-Yan Ma, Department of Critical Care Medicine, Linyi People's Hospital, 27 Jiefang Road, Linyi, Shandong 276003, P.R. China

E-mail: machengyan1012@163.com

Key words: cytomegalovirus infection, prognosis, inflammatory bowel disease, meta-analysis

\section{Introduction}

Inflammatory bowel disease (IBD) commonly describes two types of chronic intestinal disorders of the gastrointestinal tract: Ulcerative colitis (UC) and Crohn's disease (CD) (1). $\mathrm{UC}$ is characterized by diffuse inflammation of the mucosa of the colon and rectum, and has a relatively high prevalence of 5.3-63.6 per 100,000 individuals in developing areas and $37.5-238$ per 100,000 individuals in developed areas (2). CD is a disease that can occur in any part of the gastrointestinal tract, and has a growing incidence in the Asian countries (3). IBD patients are at high risk of acquiring cytomegalovirus (CMV) infection, as they are frequently treated with immunosuppressive drugs (4). These agents have moderate effects on maintaining corticosteroid-induced remission, while common effects on relapse (5). Although the etiology of IBD and the impact of antiviral treatment on the course of IBD remain poorly understood, various studies have suggested that viral infections, particularly infection with CMV, are associated with the onset and aggravation of IBD, thus causing a poor prognosis $(6,7)$.

CMV belongs to the herpesviridae family of viruses and contains a double-stranded DNA, with its effect ranging from asymptomatic infection in immunocompetent hosts to severe end-organ damage in patients with impaired cellular immunity (8). CMV often results in primary infection in humans, and later persists in a latent stage for life (9). Accumulating evidence suggested that CMV disease is more common in patients with severe IBD or steroid-refractory patients (7). CMV infection always occurs in immunocompromised hosts, and the reported prevalence of CMV infection in IBD patients is variable as the bowel is the largest immunological organ of the body (10). It is estimated that CMV infection in IBD patients is within the range of $33-36 \%$ in patients with steroid-refractory UC and $21-34 \%$ in patients suffering from severe UC (11). Colonic CMV reactivation is considered to be an exacerbating factor in patients with UC and those refractory to immunosuppressive therapies due to the poor prognosis of UC patients with concomitant CMV infection (12). Previous studies have demonstrated that local intestinal inflammation and additional immunosuppressive therapies, such as corticosteroid administration, may induce colonic CMV reactivation in IBD patients $(12,13)$. However, another study presented conflicting results on the association of CMV reactivation with 
the occurrence or severity of IBD exacerbation (1). Therefore, the aim of the present study was to investigate the effect of CMV infection on the prognosis of IBD using data collected from previous relevant studies to perform a meta-analysis.

\section{Materials and methods}

Literature search. The literature was comprehensively screened and eligible studies were collected to examine the effects of CMV infection on the prognosis of IBD. The following computerized databases were screened: Springerlink (link.springer.com),PubMed(www.ncbi.nlm.nih.gov/pubmed), Web of Science (wok.mimas.ac.uk), Wiley Online Library (onlinelibrary.wiley.com), Chinese Biomedical Database (http://www.sinomed.ac.cn/zh/), China National Knowledge Infrastructure (www.cnki.net), Wanfang database (www.wanfangdata.com) and the VIP database (www.cqvip.com). Additional pertinent studies were obtained by quadratic search. The search included studies published until September 2014. A combination of keywords and free words was applied in the process of collecting literature with a highly efficient and sensitive searching strategy. The search involved a CMV keyword ('cytomegalovirus' or 'cytomegaloviruses' or 'salivary gland viruses' or 'viruses, salivary gland' or 'herpesvirus 5, human') and an IBM keyword ('inflammatory bowel diseases' or 'inflammatory bowel disease' or 'bowel diseases, inflammatory').

Inclusion and exclusion criteria. Eligible studies were selected according to the following inclusion criteria: i) Clinical cohort studies; ii) subjects in the enrolled studies had a confirmed diagnosis of IBD based on clinical, radiologic, endoscopic and histologic parameters (14); iii) the detection method used was polymerase chain reaction or enzyme-linked immunosorbent assay; iv) the outcome index included the progress of CMV-positive and CMV-negative patients with IBD, as well as the effects of glucocorticoid treatment, colectomy rate, prevalence of severe IBD and the disease extent; and v) the language of selected studies was restricted to Chinese and English. The corresponding exclusion criteria were: i) Studies containing summary and abstracts only; ii) studies with insufficient information; iii) duplicated publications; and iv) unpublished studies.

Data extraction and quality assessment. The data from eligible studies were extracted by two separated investigators. Relevant information was collected as follows: First author, publication year, study design, country of study and patient ethnicity, patient gender and age, effects of glucocorticoid treatment, disease duration, colectomy rate, severity degree of diseases and disease extent. Subsequently, the two investigators independently assessed the methodological quality of the included cohort trials using the Newcastle-Ottawa Scale (NOS) criteria (15). The NOS criteria associated with the selection of the cohort were as follows: Representativeness of the exposed cohort (NOS1); selection of the non-exposed cohort (NOS2); ascertainment of exposure (NOS3); and demonstration that an outcome of interest was not present at the beginning of the study (NOS4). In addition, NOS criteria involving the comparability of the cohorts were as follows: The study was selected and analyzed according to the most important factor (NOS5); and the study controlled other confounding factors (NOS6). Finally, the following NOS criteria involved the assessment of the outcome: Sufficiently long follow-up for outcome to occur (NOR8); and adequacy of cohort follow-up (NOR9). A third investigator was prepared to resolve any disagreement on the inclusion of a single study.

Statistical analysis. Stata 12.0 software (Stata Corp., College Station, TX, USA) was used for statistical analysis. The fixed effects model or random effects model was adopted for the calculation of the relative risk (RR) and standardized mean difference (SMD), along with the 95\% confidence interval (95\% CI). These statistical models aimed to evaluate the prognosis of patients with IBD in association with the effects of corticosteroid therapy, colectomy ratio, prevalence of severe IBD and disease extent. Z-test was employed to detect the significance of the pooled effect size $(15,16)$. In addition, Cochran's Q-test (in which differences with $\mathrm{P}<0.05$ were considered as statistically significant) and $\mathrm{I}^{2}$ tests were employed to quantify heterogeneity among the parameters of the included trials (17). When $\mathrm{P}<0.05$ or $\mathrm{I}^{2}>50 \%$ were obtained, which indicated heterogeneous results, the random effects model was conducted; otherwise, the fixed effects model was implemented. In order to evaluate the influence of single study on the overall estimate, a sensitivity analysis was employed according to the Cochrane Handbook for Systematic Reviews of Interventions (http://handbook.cochrane.org/). Furthermore, for the purpose of ensuring the reliability of the results, publication bias was examined by funnel plots and Egger's linear regression test (with $\mathrm{P}<0.05$ indicating a statistically significant difference) $(18,19)$.

\section{Results}

Baseline characteristics of eligible studies. Initially, a total of 195 studies (28 in Chinese and 167 in English) were identified from the literature screening, including 193 from electronic search and 2 from manual search. Subsequently, 142 articles were excluded due to not meeting the inclusion criteria following review of the title and abstract, and 46 full-text articles remained. Ultimately, 7 studies (6 in English and 1 in Chinese) satisfied the inclusion criteria after elimination of 36 off-topic studies and 3 studies with insufficient information. The enrolled studies $(1,12,13,20-23)$ were published between 2001 and 2013, and provided complete clinical data for 374 patients with IBD. Among these 7 studies, 5 studies included Asian patients and 2 studies included Caucasian patients. One was conducted in China, 3 were conducted in Japan, 1 was conducted in India, 1 was conducted in France and 1 was conducted in Italy. Furthermore, the 7 studies contained 374 patients with IBD; of these, 104 cases were CMV-positive and 270 cases were CMV-negative. The baseline characteristics and NOS scores for the 7 eligible studies are summarized in Table I and Fig. 1, respectively.

Association of disease duration with CMV infection. Of the studies included in the meta-analysis, 3 reported the disease duration of CMV-positive and CMV-negative patients $(12,20,23)$. Heterogeneity was observed $\left(\mathrm{I}^{2}, 95.8 \% ; \mathrm{P}_{\mathrm{h}}<0.001\right)$ and the 
Table I. Baseline characteristics for the eligible studies.

\begin{tabular}{|c|c|c|c|c|c|c|c|c|c|c|c|c|}
\hline \multirow{2}{*}{$\begin{array}{l}\text { First } \\
\text { author }\end{array}$} & \multirow[b]{2}{*}{ Year } & \multirow[b]{2}{*}{ Country } & \multirow[b]{2}{*}{ Ethnicity } & \multirow{2}{*}{$\begin{array}{c}\text { Study } \\
\text { language }\end{array}$} & \multirow[b]{2}{*}{$\mathrm{N}$} & \multicolumn{2}{|c|}{ Patients } & \multicolumn{2}{|c|}{ Gender $(\mathrm{M} / \mathrm{F})$} & \multicolumn{2}{|c|}{ Age (years) } & \multirow[b]{2}{*}{ Ref. } \\
\hline & & & & & & $\mathrm{CMV+}$ & CMV- & $\mathrm{CMV+}$ & CMV- & $\mathrm{CMV}+$ & CMV- & \\
\hline Zhao & 2011 & China & Asian & Chinese & 76 & 21 & 55 & $13 / 8$ & $32 / 23$ & $46.2 \pm 19$ & $47.6 \pm 19.2$ & (23) \\
\hline Yoshino & 2007 & Japan & Asian & English & 30 & 17 & 13 & $14 / 16$ & - & $40.8 \pm 17.6$ & - & (22) \\
\hline Kishore & 2004 & India & Asian & English & 63 & 10 & 53 & $2 / 8$ & $30 / 23$ & 39 & 36 & (20) \\
\hline Leveque & 2010 & France & Caucasian & English & 53 & 7 & 46 & $23 / 30$ & - & 38.5 & - & (1) \\
\hline Cottone & 2001 & Italy & Caucasian & English & 19 & 7 & 12 & - & - & 48.5 & 35.8 & (21) \\
\hline Fukuchi & 2013 & Japan & Asian & English & 51 & 15 & 36 & $7 / 8$ & $20 / 16$ & $42.9 \pm 3.8$ & $36.2 \pm 2.4$ & (12) \\
\hline Iida & 2013 & Japan & Asian & English & 82 & 27 & 55 & $15 / 12$ & $32 / 23$ & 43.2 & 38.9 & (13) \\
\hline
\end{tabular}

CMV, cytomegalovirus; $\mathrm{N}$, total number of patients; $\mathrm{M}$, male; F, female.

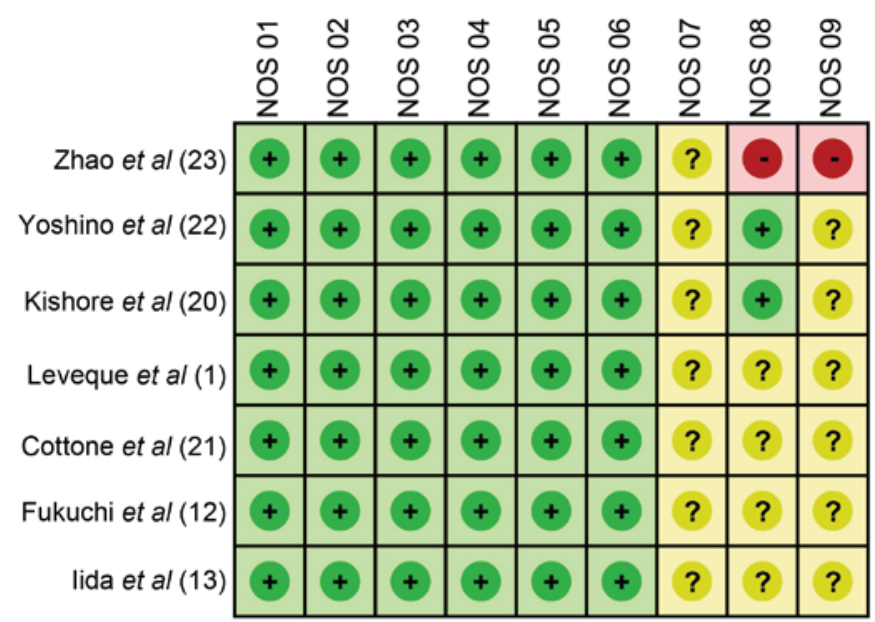

Figure 1. Assessment of the methodological quality of the 7 eligible studies according to the NOS scores. NOS, Newcastle-Ottawa scale; +, yes; -, no; ?, unclear.

random effect model was applied to compare the observations of the studies. According to the meta-analysis results, the disease duration of CMV-positive patients was significantly reduced when compared with that of CMV-negative patients (SMD, $-0.81 ; 95 \%$ CI, -1.19 to $-0.43 ; \mathrm{P}<0.001)$, and the forest plot of this analysis is shown in Fig. 2A. The results show that CMV infection may be associated with the disease duration of IBD.

Association of corticosteroid therapy efficacy with CMV infection. A total of 4 studies reported the efficacy of corticosteroid therapy in both CMV-positive and CMV-negative patients $(1,20,22,23)$. No heterogeneity was observed among the 4 studies $\left(\mathrm{I}^{2}, 32.1 \% ; \mathrm{P}_{\mathrm{h}}=0.220\right)$, and thus the fixed effect model was applied. As shown in Fig. 2B, the results of the present meta-analysis demonstrated that corticosteroid therapy had a markedly improved efficacy in CMV-positive patients compared with their efficacy in CMV-negative patients (RR, 1.24; $95 \% \mathrm{CI}$, 1.02-1.49; $\mathrm{P}=0.029)$. The results show that $\mathrm{CMV}$ infection may be associated with the corticosteroid therapy efficacy of IBD.

Association of ratio of patients undergoing colectomy with CMV infection. Out of the 7 eligible studies, 4 studies reported the colectomy rate in both CMV-positive and CMV-negative patients $(1,20,22,23)$. No heterogeneity was observed $\left(\mathrm{I}^{2}, 32.8 \% ; \mathrm{P}_{\mathrm{h}}=0.216\right)$ and the fixed effect model was applied to examine the association of colectomy rate with CMV infection. As shown in Fig. 2C, the findings of the present study suggested that a significantly higher rate of CMV-positive patients received colectomy, when compared with CMV-negative patients (RR, 2.13; 95\% CI, 1.03-4.40; $\mathrm{P}=0.042$ ). The results show that $\mathrm{CMV}$ infection may be associated with the colectomy rate in IBD patients.

Association of severe IBD incidence with CMV infection. In total, 3 studies reported the incidence of severe IBD in both CMV-positive and CMV-negative patients $(1,20,23)$. No heterogeneity was observed $\left(\mathrm{I}^{2}, 45.8 \% ; \mathrm{P}_{\mathrm{h}}=0.158\right)$ and the fixed effect model was used to perform meta-analysis. The results identified that the incidence of severe IBD in CMV-positive patients was significantly higher compared with that in CMV-negative patients (RR, 1.32; 95\% CI, 1.04-1.67; P=0.022; Fig. 2D). The results show that the $\mathrm{CMV}$ infection may be associated with the incidence of severe IBD.

Association of disease location with CMV infection. The association of the area to which IBD extended with CMV infection was also investigated. In total 5 of the eligible studies reported the presence of pancolitis $(1,12,20,22,23)$ and 5 studies reported left-sided IBD $(1,12,13,21,22)$ in both CMV-positive and CMV-negative patients. No heterogeneity was observed $\left(\mathrm{I}^{2}<0.01 \% ; \mathrm{P}_{\mathrm{h}}=0.602\right)$ and the fixed effect model was applied for meta-analysis. Considering the IBD onset area, CMV-positive patients were found to present significantly higher susceptibility to pancolitis compared with the CMV-negative patients (RR, 1.31; 95\% CI, 1.01-1.72; P=0.045; Fig. 2E). By contrast, no significant difference was identified in the susceptibility of CMV-positive or CMV-negative patients to the incidence of left-sided IBD (RR, 0.97; 95\% CI, 0.72-1.30; P=0.828; Fig. 2F). The results show that $\mathrm{CMV}$ infection may be associated with the disease location of IBD.

Sensitivity analysis and publication bias investigation. The results of sensitivity analysis demonstrated that no single study was able to impact the overall estimate of the SMD 
A

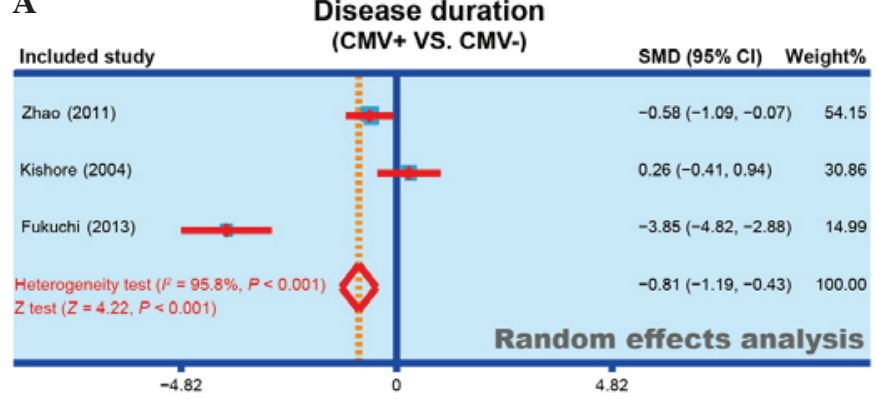

C

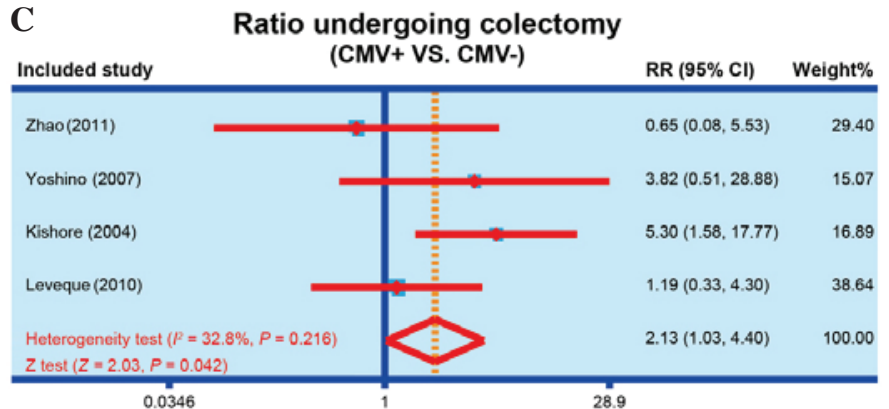

$\mathbf{E}$

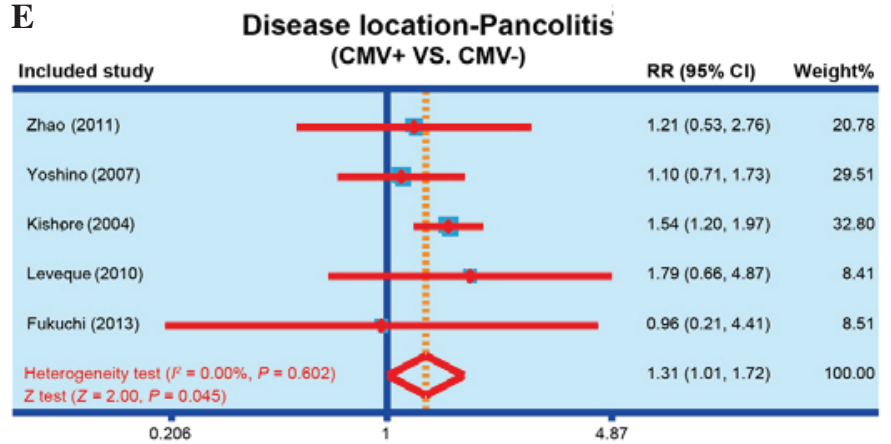

B

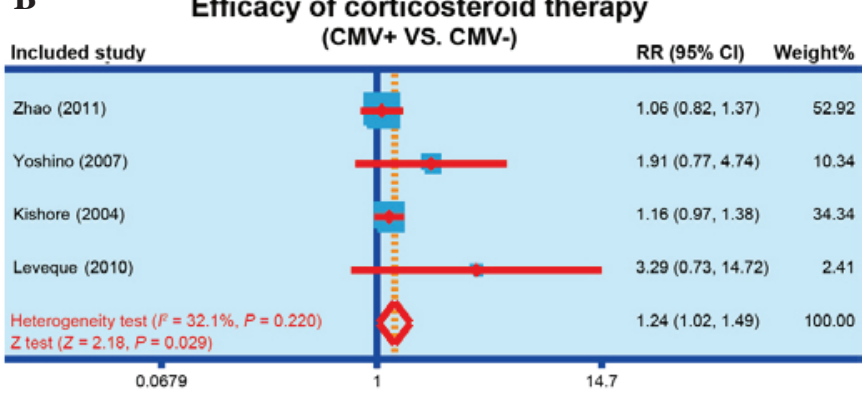

D

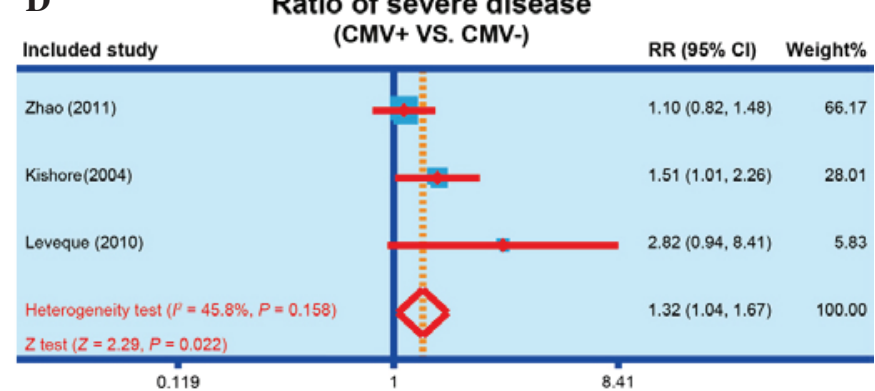

F

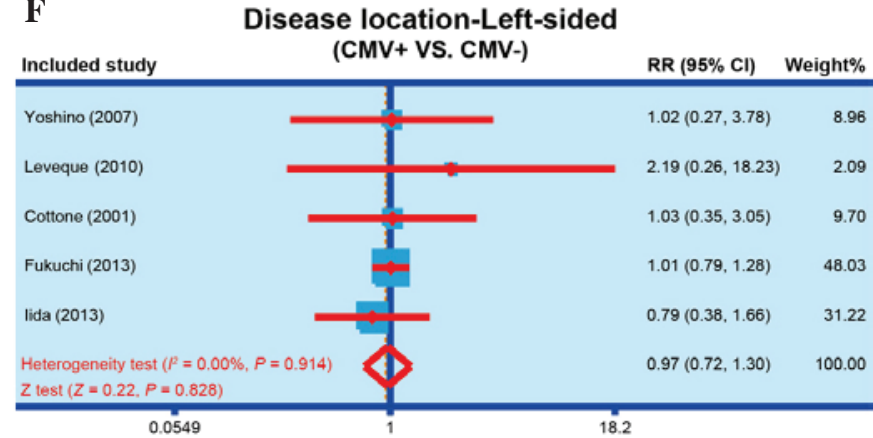

Figure 2. Forest plots obtained by random effects model analysis, performed to compare the (A) disease duration of IBD, (B) efficacy of corticosteroid therapy, (C) colectomy rate, (D) incidence of severe IBD, (E) incidence of pancolitis and (F) incidence of life-sided IBD, between the CMV-positive and CMV-negative patients. IBD, inflammatory bowel disease; CMV, cytomegalovirus; SMD, standardized mean difference; RR, relative risk; $95 \%$ CI, 95\% confidence interval.

value of disease duration in the CMV-positive or -negative patients (Fig. 3A). In addition, the RR value of efficacy of corticosteroid therapy, colectomy rate, the incidence of severe IBD and disease location were also not affected by a single study (Fig. 3B-F). Furthermore, publication bias was investigated using funnel plots and Egger's linear regression. As shown in Fig. 4, no evident asymmetry was observed in the shapes of the funnel plots, which appeared to be symmetric, while the Egger's regression test suggested the absence of publication bias. These results suggested that no significant publication bias was detected in the present meta-analysis $(\mathrm{P}>0.05)$.

\section{Discussion}

In the present study, the data of 374 patients suffering from deterioration of IBD were analyzed retrospectively. According to the results, the current study suggests that CMV infection may be an important biomarker in determining the prognosis of IBD. The human response to CMV infection is complex and encompasses multiple aspects of the human immune system (24). Although the role of CMV in active IBD has been controversial, early studies have highlighted the association of CMV infection with severe active IBD and high doses of immunosuppressive medication $(10,25)$.

The findings of the present study demonstrated that CMV infection is potentially associated with the disease duration, efficacy of corticosteroid therapy, colectomy rate, the incidence of severe IBD and the disease location in patients with IBD. CMV may present in immunosuppressed patients due to reactivation of a latent infection, and it has a detrimental effect on the disease severity and outcome of IBD patients (26). Increased CMV reactivity has been demonstrated to be associated with active IBD disease and longer disease duration (11). Due to the association of CMV with severe steroid-refractory IBD, CMV can be involved in flares of IBD by causing CMV colitis directly or by exacerbating underlying IBD (10). It has been observed that the prevalence of CMV infection in active UC patients is lower than that previously reported in patients with acute severe colitis and steroid-refractory colitis, suggesting that the CMV infection is correlated with the incidence of severe IBD (27). It is suggested that patients with steroid-refractory or steroid-dependent IBD should be screened for CMV infection prior to increasing the dose and number of immunosuppressive drugs administrated $(27,28)$. 
A

Disease duration

(CMV+ VS. CMV-)

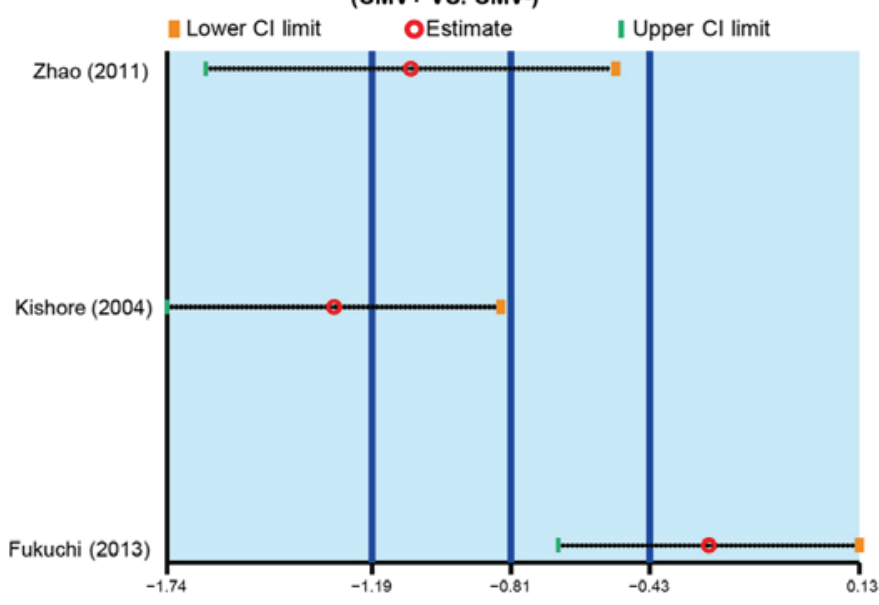

C

Ratio undergoing colectomy (CMV+ VS. CMV-)

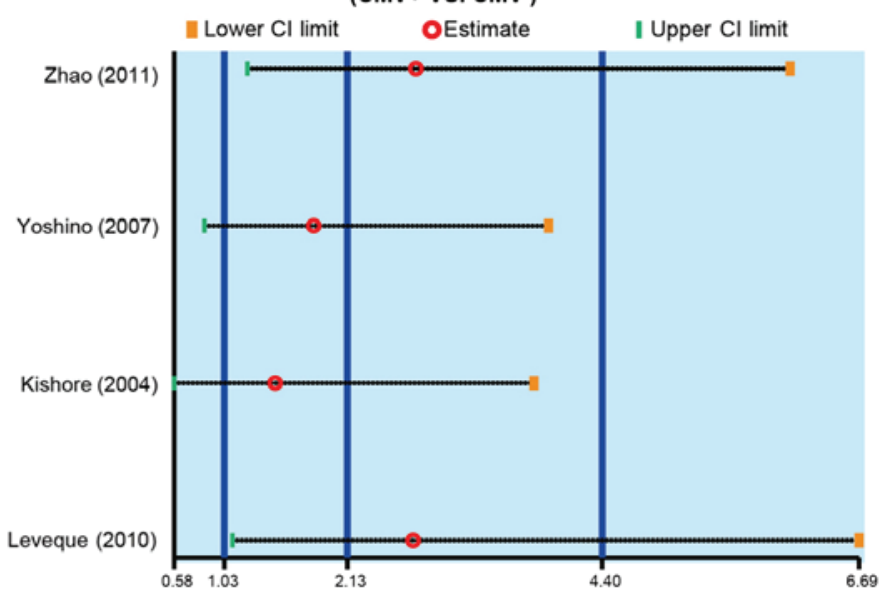

E

Disease location-Pancolitis (CMV+ VS. CMV-)

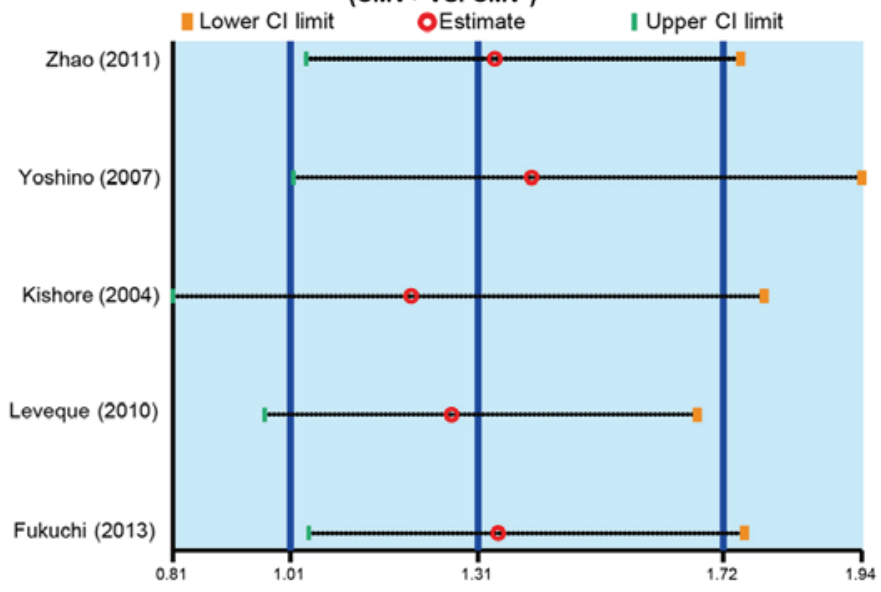

B

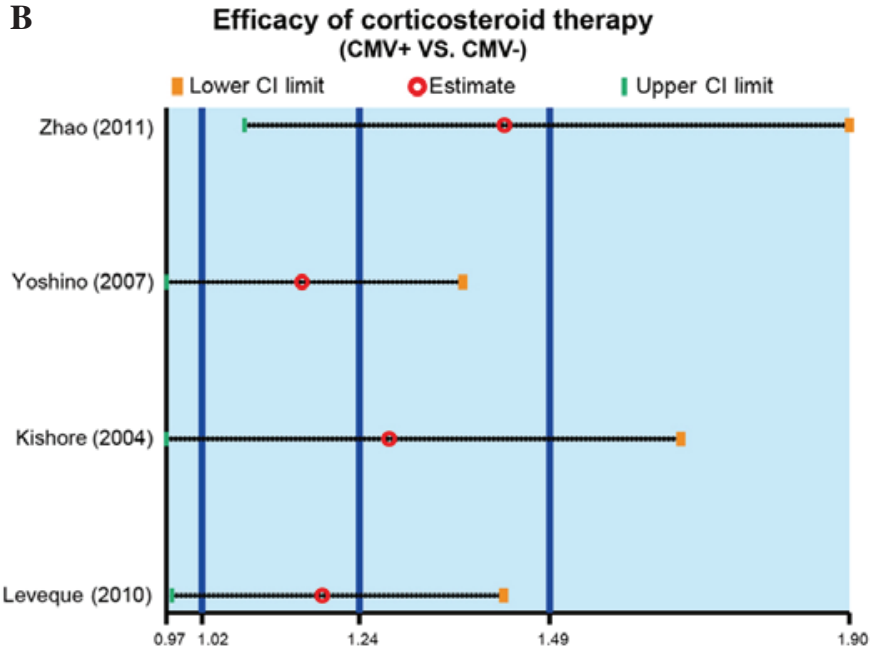

D

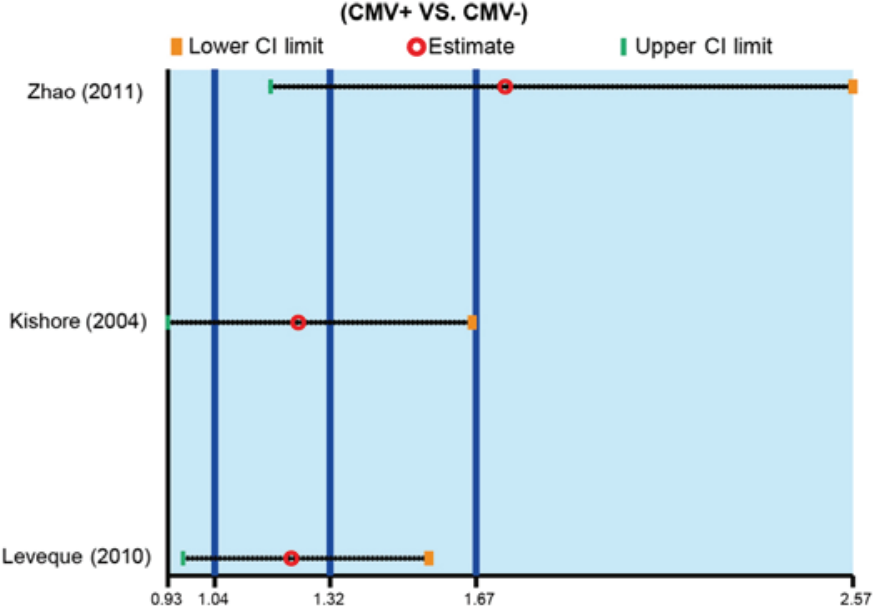

F

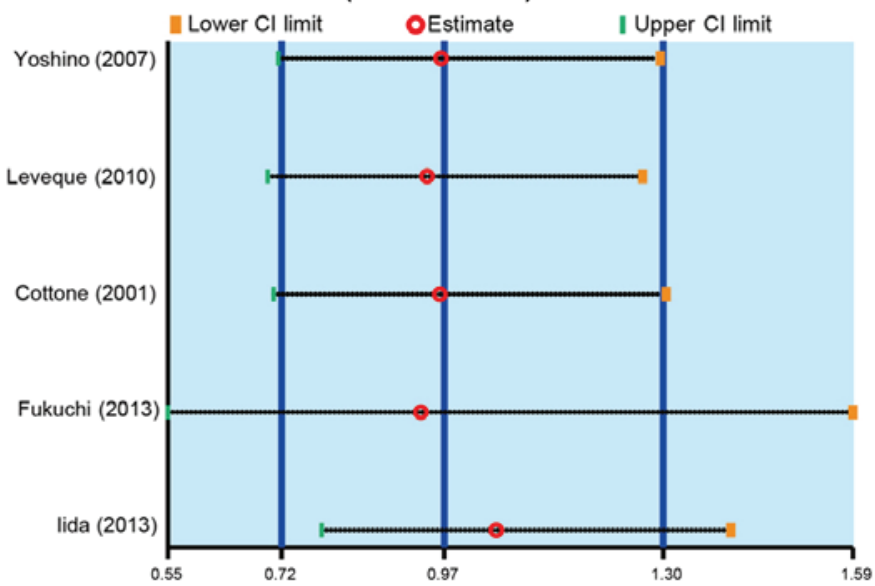

Figure 3. Sensitivity analysis performed based on the (A) disease duration of IBD, (B) efficacy of corticosteroid therapy, (C) colectomy rate, (D) incidence of severe IBD, (E) incidence of pancolitis and (F) incidence of left-sided IBD, in CMV-positive and CMV-negative patients. IBD, inflammatory bowel disease; CMV, cytomegalovirus; CI, confidence interval.

The findings of Leveque et al (1) also demonstrates a significant association between the treatment with high-dose systemic corticosteroids and the detection of CMV in IBD patients. Furthermore, CMV is present in its latent form in the majority of healthy subjects, so it is important to know the disease location for better treatment of IBD patients. In the present meta-analysis, disease location data demonstrated that the prevalence of pancolitis may be affected by the presence of CMV infection in the IBD patient. In general, CMV infection results to severe IBD or recovery of IBD, and thus it may be a useful biomarker in the determination of prognosis in IBD patients. 
A

Disease duration

(CMV+ VS. CMV-)

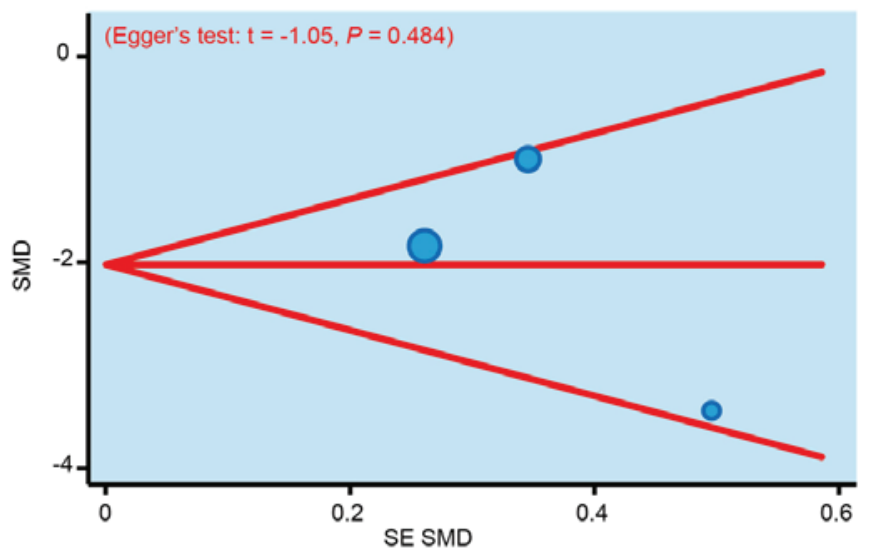

C

Ratio undergoing colectomy (CMV+ VS. CMV-)

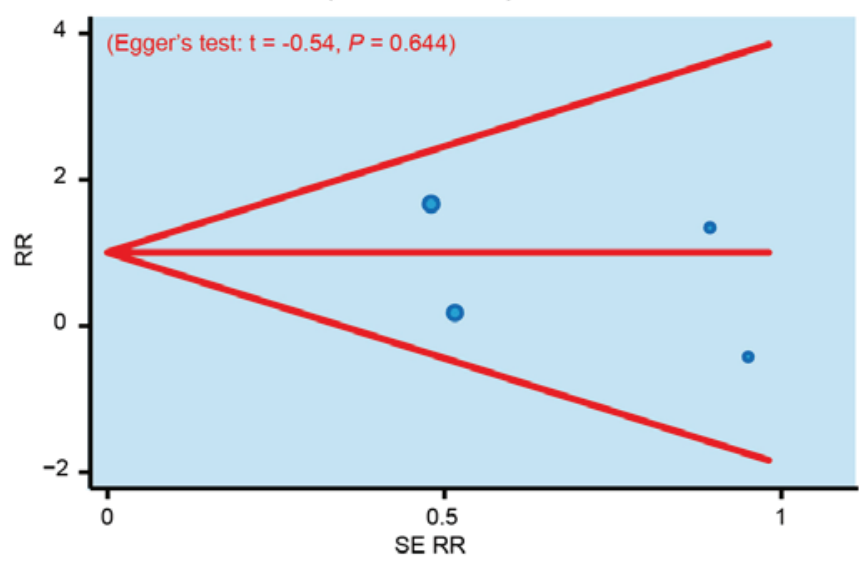

E

Disease location-Pancolitis (CMV+ VS. CMV-)

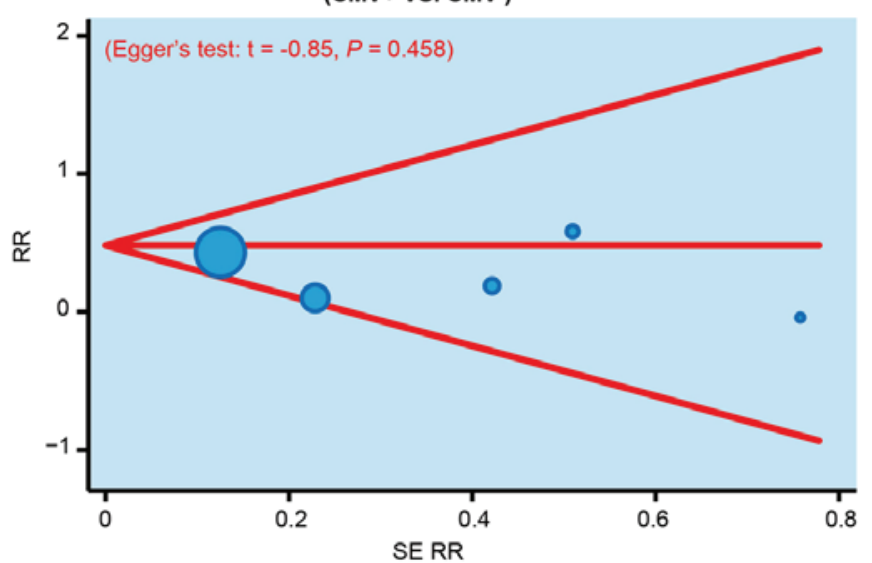

B Efficacy of corticosteroid therapy (CMV+ VS. CMV-)

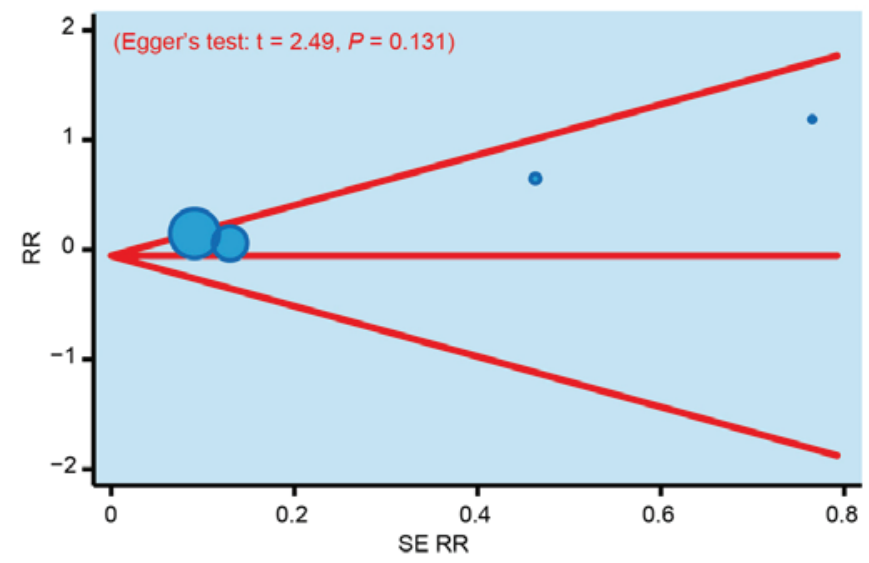

D

Ratio of severe disease (CMV+ VS. CMV-)

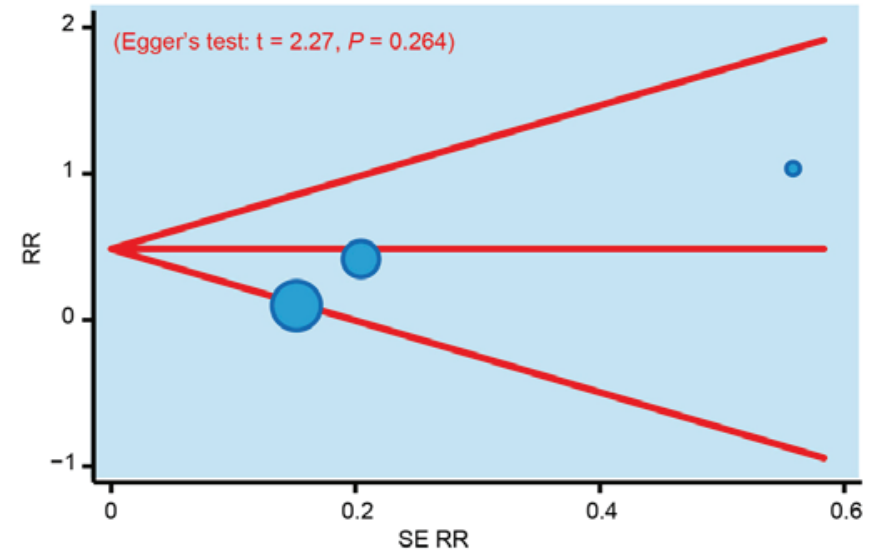

$\mathbf{F}$

Disease location-Left-sided (CMV+ VS. CMV-)

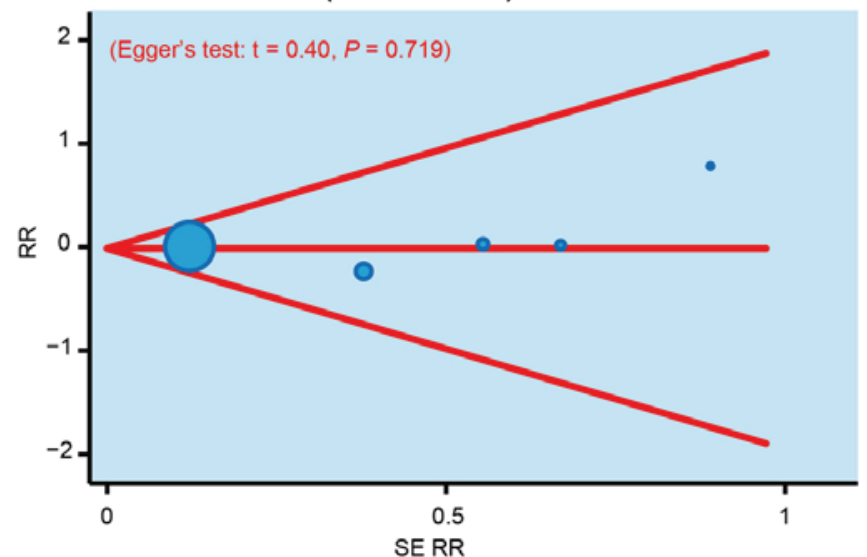

Figure 4. Funnel plots and Egger's linear regression tests were performed to investigate the publication bias in the present meta-analysis, based on the (A) disease duration of IBD, (B) efficacy of corticosteroid therapy, (C) colectomy rate, (D) incidence of severe IBD, (E) incidence of pancolitis (disease location), and (F) the incidence of left-sided IBD (disease location), in CMV-positive and CMV-negative patients. IBD, inflammatory bowel disease; CMV, cytomegalovirus; SMD, standardized mean difference; SE RR, standard error of relative risk.

However, certain limitations of the present meta-analysis should be noted. Firstly, due to the limited number of IBD patients in the enrolled studies, the sample size in each subgroup analysis was rather small, which may decrease the accuracy of this meta-analysis. In addition, all included studies were published in English or Chinese in the various electronic databases searched, which may bias the results, since other languages or unpublished studies were not considered.

In conclusion, the present meta-analysis revealed that CMV infection is strongly correlated with IBD prognosis. The findings demonstrated that CMV infection may be associated with the disease duration of IBD, efficacy of corticosteroid therapy, colectomy rate, the incidence of severe IBD and 
disease location. Therefore, CMV infection is suggested to be a biomarker of IBD prognosis, and determining the presence of CMV infection in IBD patients may assist in the selection of an efficient treatment strategy.

\section{Acknowledgements}

The authors would like to thank their colleagues for their valuable advice and comments on the present study.

\section{References}

1. Leveque N, Brixi-Benmansour H, Reig T, Renois F, Talmud D, Brodard V, Coste JF, De Champs C, Andréoletti L and Diebold MD: Low frequency of cytomegalovirus infection during exacerbations of inflammatory bowel diseases. J Med Virol 82: 1694-1700, 2010.

2. Da Silva BC, Lyra AC, Rocha R and Santana GO: Epidemiology, demographic characteristics and prognostic predictors of ulcerative colitis. World J Gastroenterol 20: 9458-9467, 2014.

3. Lee KM and Lee JM: Crohn's disease in Korea: Past, present, and future. Korean J Intern Med 29: 558-570, 2014.

4. D'Ovidio V, Vernia P, Gentile G, Capobianchi A, Marcheggiano A, Viscido A, Martino P and Caprilli R: Cytomegalovirus infection in inflammatory bowel disease patients undergoing anti-TNFalpha therapy. J Clin Virol 43: 180-183, 2008.

5. Lichtenstein GR, Rutgeerts P,Sandborn WJ, Sands BE,Diamond RH, Blank M, Montello J, Tang L, Cornillie F and Colombel JF: A pooled analysis of infections, malignancy, and mortality in infliximab- and immunomodulator-treated adult patients with inflammatory bowel disease. Am J Gastroenterol 107: 1051-1063, 2012.

6. Delvincourt M, Lopez A, Pillet S, Bourrier A, Seksik P, Cosnes J, Carrat F, Gozlan J, Beaugerie L, Roblin X, et al: The impact of cytomegalovirus reactivation and its treatment on the course of inflammatory bowel disease. Aliment Pharmacol Ther 39: 712-720, 2014.

7. Jones A, McCurdy JD, Loftus EV Jr, Bruining DH, Enders FT, Killian JM and Smyrk TC: Effects of antiviral therapy for patients with inflammatory bowel disease and a positive intestinal biopsy for cytomegalovirus. Clin Gastroenterol Hepatol 13: 949-955, 2015.

8. McCurdy JD, Jones A, Enders FT, et al: A model for identifying cytomegalovirus in patients with inflammatory bowel disease. Clin Gastroenterol Hepatol 13: 131-137; quiz e7, 2015.

9. Garrido E, Carrera E, Manzano R and Lopez-Sanroman A: Clinical significance of cytomegalovirus infection in patients with inflammatory bowel disease. World J Gastroenterol 19: 17-25, 2013.

10. Kim JJ, Simpson N, Klipfel N, Debose R, Barr N and Laine L: Cytomegalovirus infection in patients with active inflammatory bowel disease. Dig Dis Sci 55: 1059-1065, 2010.

11. Nowacki TM, Bettenworth D, Ross M, Heidemann J, Lehmann PV and Lügering A: Cytomegalovirus (CMV)-specific perforin and granzyme B ELISPOT assays detect reactivation of CMV infection in inflammatory bowel disease. Cells 1: 35-50, 2012.

12. Fukuchi T, Nakase H, Matsuura M, Yoshino T, Toyonaga T, Ohmori K, Ubukata S, Ueda A, Eguchi T, Yamashita H, et al: Effect of intensive granulocyte and monocyte adsorptive apheresis in patients with ulcerative colitis positive for cytomegalovirus. J Crohns Colitis 7: 803-811, 2013.
13. Iida T, Ikeya K, Watanabe F, Abe J, Maruyama Y, Ohata A, Teruyuki S, Sugimoto K and Hanai H: Looking for endoscopic features of cytomegalovirus colitis: A study of 187 patients with active ulcerative colitis, positive and negative for cytomegalovirus. Inflamm Bowel Dis 19: 1156-1163, 2013.

14. Dubinsky M and Braun J: Diagnostic and prognostic microbial biomarkers in inflammatory bowel diseases. Gastroenterology 149: 1265.e3-1274.e3, 2015.

15. Stang A: Critical evaluation of the Newcastle-Ottawa scale for the assessment of the quality of nonrandomized studies in meta-analyses. Eur J Epidemiol 25: 603-605, 2010.

16. Chen H, Manning AK and Dupuis J: A method of moments estimator for random effect multivariate meta-analysis. Biometrics 68: 1278-1284, 2012.

17. Zintzaras E and Ioannidis JP: HEGESMA: Genome search meta-analysis and heterogeneity testing. Bioinformatics 21: 3672-3673, 2005.

18. Song F and Gilbody S: Bias in meta-analysis detected by a simple, graphical test. Increase in studies of publication bias coincided with increasing use of meta-analysis. BMJ 316: 471, 1998.

19. Peters JL, Sutton AJ, Jones DR, Abrams KR and Rushton L: Comparison of two methods to detect publication bias in meta-analysis. JAMA 295: 676-680, 2006.

20. Kishore J, Ghoshal U, Ghoshal UC, Krishnani N, Kumar S, Singh M and Ayyagari A: Infection with cytomegalovirus in patients with inflammatory bowel disease: Prevalence, clinical significance and outcome. J Med Microbiol 53: 1155-1160, 2004.

21. Cottone M, Pietrosi G, Martorana G, Casà A, Pecoraro G, Oliva L, Orlando A, Rosselli M, Rizzo A and Pagliaro L: Prevalence of cytomegalovirus infection in severe refractory ulcerative and Crohn's colitis. Am J Gastroenterol 96: 773-775, 2001.

22. Yoshino T, Nakase H, Ueno S, Uza N, Inoue S, Mikami S, Matsuura M, Ohmori K, Sakurai T, Nagayama S, et al: Usefulness of quantitative real-time PCR assay for early detection of cytomegalovirus infection in patients with ulcerative colitis refractory to immunosuppressive therapies. Inflamm Bowel Dis 13: 1516-1521, 2007.

23. Zhao K, Li ZM, Zuo YN, Xie ZX, Li LK, Zhang B and Peng D: Correlation of cytomegalovirus infection in the intestine of patients with inflammatory bowel disease. Zhong Guo Yi Yao Dao Kan 13: 1863-1865, 2011 (In Chinese).

24. Miller-Kittrell M and Sparer TE: Feeling manipulated: Cytomegalovirus immune manipulation. Virol J 6: 4, 2009.

25. Pillet S, Pozzetto B, Jarlot C, Paul S and Roblin X: Management of cytomegalovirus infection in inflammatory bowel diseases. Dig Liver Dis 44: 541-548, 2012.

26. Maconi G, Lombardini M, Furfaro F, Bezzio C, Zerbi P and Ardizzone S: Long-term outcome of inflammatory bowel diseases with cytomegalovirus colitis: Effect of antiviral treatment. Eur J Gastroenterol Hepatol 26: 1146-1151, 2014.

27. Osaki R, Andoh A, Tsujikawa T, Ogawa A, Koizumi Y, Nakahara T, Hata K, Sasaki M, Saito Y and Fujiyama Y: Acute cytomegalovirus infection superimposed on corticosteroid-naïve ulcerative colitis. Intern Med 47: 1341-1344, 2008.

28. Domenech E, Vega R, Ojanguren I, Hernández A, Garcia-Planella E, Bernal I, Rosinach M, Boix J, Cabré E and Gassull MA: Cytomegalovirus infection in ulcerative colitis: A prospective, comparative study on prevalence and diagnostic strategy. Inflamm Bowel Dis 14: 1373-1379, 2008. 